Instituto Internacional de Investigación y Desarrollo Tecnológico Educativo INDTEC, C.A.

DOI: https://doi.org/10.29394/scientific.issn.2542-2987.2017.2.6.7.134-153

OAI-PMH: http://www.indteca.com/ojs/index.php/Revista Scientific/oai

\title{
Acciones Ambientalistas para la Conservación de las Cuencas Hidrográficas
}

\author{
Autora: Jeineth del Carmen Navea Rojas \\ Universidad Pedagógica Experimental Libertador, UPEL \\ njeinethdelc@gmail.com \\ Barinas, Venezuela
}

\section{Resumen}

El presente estudio tiene como propósito ejecutar acciones ambientalistas para la conservación de la cuencas hidrográficas que sensibilicen a los habitantes del sector "Las Dos Bocas" aledaño a la cuenca media alta del rio La Acequia, perteneciente a la parroquia Ciudad Bolivia, municipio Pedraza, estado Barinas, en la conservación de su caudal como recurso hídrico indispensable para los pedraceños, evitando perturbaciones de origen antropogénico, como la deforestación y quema de vegetación en zonas ribereñas. Esta investigación responde a una naturaleza basada en un paradigma cualitativo, bajo un diseño de investigación acción participativa y se complementa su enfoque metodológico con el uso de las técnicas de observación y la entrevista no estructurada. Como conclusiones se obtuvo que se amerita de acciones ambientalistas que realmente proporcionen los conocimientos, la sensibilización y el compromiso de la comunidad para generar cambios de actitud y comportamientos, en función de la valoración del ambiente como un elemento interviniente para lograr la calidad de vida; por lo cual estas requieren atender tres aspectos o fases: Sensibilización, capacitación e intercambio de experiencias a través de jornadas de motivación, talleres de orientación, jornadas de limpieza, prácticas de reciclaje, siembra de plantas, entre otros.

Palabras clave: acciones ambientalistas; conservación ambiental; cuencas hidrográficas. 


\title{
Environmental Actions for the Conservation of Hydrographic Basins
}

\begin{abstract}
The present study aims to implement environmental actions for the conservation of watersheds that sensitize the inhabitants of the sector "Las Dos Bocas" adjacent to the upper middle basin of the river La Acequia, belonging to the parish Ciudad Bolivia, Pedraza municipality, state Barinas, in the conservation of its flow as an essential water resource for the pedraceños, avoiding disturbances of anthropogenic origin, such as deforestation and burning of vegetation in riparian areas. This research responds to a nature based on a qualitative paradigm, under a participatory action research design and complements its methodological approach with the use of observation techniques and unstructured interview. As conclusions it was obtained that it deserves environmental actions that really provide the knowledge, awareness and commitment of the community to generate changes in attitude and behaviors, depending on the valuation of the environment as an intervening element to achieve quality of life; So they need to address three aspects or phases: Awareness raising, training and exchange of experiences through motivation days, orientation workshops, cleaning days, recycling practices, planting, among others.
\end{abstract}

Keywords: environmental actions; environmental conservation; watersheds. 


\section{Introducción}

En la actualidad el manejo integral de las cuencas es muy importante dado que genera afluentes que benefician al ser humano, por ello, es preciso que sean ampliamente tratadas y protegidas, pues de ellas depende el abastecimiento de agua limpia y suficiente, además, manejar la cuenca significa planificar todas las actividades agropecuarias y urbanas de tal manera que afecten en el menor grado posible estos importantes recursos, como lo son los ríos, hábitat para flora y fauna, protección al suelo, mejoramiento de la calidad del aire. Debido a lo expuesto, Benetti y Garrido (2012), manifiestan:

Los ecosistemas fluviales y sus cuencas han estado sometidos a profundos cambios como consecuencia de perturbaciones de origen antropogénico, debido principalmente al aumento de la población humana y consiguiente degradación de los recursos naturales, por ello la protección de estos ecosistemas es fundamental para la conservación de sus afluentes, ya que la alteración de su estructura contamina el agua de los ríos, así como baja su corriente y por ende la vigorosidad de la corriente. (pág. 115).

Siguiendo con el orden de ideas, el manejo integral de las cuencas de los ríos son de vital importancia para la existencia de todos los seres vivos, porque genera afluentes que benefician al ser humano, además, son corrientes de agua esenciales para los ecosistemas terrestres, por ello, la humanidad ha centrado su evolución en las riberas de los mismos, debido a que poseen una enorme fertilidad, no solo desde el punto de vista agrícola, sino también desde la biodiversidad, en donde, algunos de ellos son verdaderos vergeles de vida como pueden ser el rio Nilo o en Amazonas, lugares que reúnen una gran concentración de biodiversidad.

Entre tanto, según Quagliotti, citado en Lizcano (2017), "Los espacios geográficos donde existen reservas de este líquido, comienzan a constituirse en referentes fundamentales a ser controlados para tener el dominio sobre los recursos hídricos dada su escasez, producto del cambio climático generado 
por el calentamiento global." (pág. 81). De allí, el llamado impostergable a la toma de conciencia para la preservación y cuido de los cauces naturales del planeta que garanticen la vida de las diferentes especies.

Desde esta perspectiva, la conservación de los recursos naturales es importante para ser considerada iniciativa de desarrollo sostenible, puesto que, el manejo y conservación del agua es de especial importancia, dado que se ha evidenciado una creciente carencia de agua para propósitos de consumo humano e irrigación, atribuyéndosele a la deforestación continua y la contaminación de las cuencas hidrográficas que almacenan y producen agua en las tierras altas, notándose que los efectos nocivos de la contaminación son a causa de todos aquellos agentes externos residuales dejados en las riberas de los mismos. En este sentido, Rodríguez (2013a), dice:

En los alrededores de las cuencas hidrográficas se observa incremento de la población en sus riberas, el creciente grado de industrialización, y de acuerdo con estudios realizados, se ha evaluado el grado de contaminación por materiales no degradables que terminan en los ríos y causan un daño ecológico casi irreversible, pues se ha de tardar muchos años en volver a tomar la normalidad. (pág. 25).

Cabe destacar, que Venezuela posee una particularidad común a la mayoría de los países tropicales, y es la gran cantidad de ríos que se encuentran sobre su territorio, con zonas montañosas en sus alrededores lo que hace que el potencial erosivo sea menor, además favorece la retención de agua durante mucho tiempo, impidiendo que en temporadas de sequía este se disipe, y a través del tiempo desaparezca, pues, es un recurso natural valiosísimo del cual se extrae gran variedad de aportes a la economía, a la sociedad y a la cultura en general, dado que, es una importante fuente de agua para las industrias agrícolas y ganaderas, especialmente en los estados Portuguesa, Apure, Barinas y Guárico, además de ser de gran utilidad en las distintas actividades domésticas. 
Aunado a esto, el municipio Pedraza es conocido como la capital de los ríos de Venezuela, pues, importantísimos ríos atraviesan su extensión territorial, permitiendo su desarrollo económico, dado que, son un atractivo turístico para los visitantes, tanto en el ámbito local, nacional e internacional, en ellos se desarrolla la práctica de deportes extremos como el rafting; así como también la pesca y la recreación por poseer ciertas características que la determinan.

No obstante, el río La Acequia con el pasar de los años ha venido perdiendo su caudal, motivado a que los sectores poblados se han ido desarrollando en la cabecera o su parte alta del río, deforestando sus alrededores para realizar actividades agrícolas y pecuarias generando acelerados procesos degenerativos de las especies forestales nativas que se presentan en los bosques naturales, tal es así que los pobladores de esta zona para ganar mayor espacio para las actividades agrícolas y pecuarias, va talando los bosques naturales iniciando con el corte de los árboles y luego realizan la quema con el propósito de obtener área cultivables; trayendo como consecuencia el empobrecimiento de los suelos, provocando la erosión de los mismos, además, de afectar directamente la cantidad de recarga hídrica que reduce la capacidad de infiltración superficial, subterránea y acuíferos.

Por lo antes expuesto, la conservación de los ríos ha estado presente desde épocas pasadas, estos movimientos conservacionistas han ido creciendo drásticamente en los últimos años, sin embargo no se han tomado muchas iniciativas y mecanismos para preservar el vital líquido, pese a ello, y debido al incontenible crecimiento demográfico, el continuo avance de la frontera agrícola, la intensa actividad de extracción de madera y la poca conciencia ambiental, los ríos, presenta un cuadro de deterioro alarmante.

En virtud a ello la presente investigación se centra en la realizar un plan acción en aras de conservar la cuenca en la parte media alta del río La Acequia en conjunto con la participación de los estudiantes del 2do año del Nivel Media, 
de la Escuela Técnica Agropecuaria Nacional (ETAN) Don Simón Rodríguez, que comprende actividades de recolección de desechos sólidos y siembra de árboles, específicamente en la parte alta del río, así como también realizar charlas de motivación y sensibilización en pro de la conservación del medio ambiente.

En atención a la situación planteada y dada la importancia que reviste la conservación de las cuencas del río La Acequia, parroquia Ciudad Bolivia, municipio Pedraza, estado Barinas, surgen las siguientes interrogantes: ¿Cuál es la necesidad de implementar acciones ambientalistas para la conservación de la cuenca media-alta del río La Acequia, parroquia Ciudad Bolivia, municipio Pedraza estado Barinas?; ¿Qué acciones ambientalistas pueden diseñarse para la conservación de la cuenca media-alta del río La Acequia?; ¿La implementación de acciones ambientalistas incentivarán a la conservación de la cuenca media-alta del río La Acequia?; ¿Qué resultados arrojaría la implementación de acciones ambientalistas para la conservación de la cuenca media-alta del río La Acequia?.

Se destaca, entre los objetivos del estudio el propósito general: Aplicar acciones ambientalistas para la conservación de la cuenca media-alta del río La Acequia, en el sector "Las Dos Bocas" parroquia Ciudad Bolivia, municipio Pedraza estado Barinas y los específicos: Diagnosticar la necesidad de implementar acciones ambientalistas para la conservación de la cuenca media-alta del río La Acequia, parroquia Ciudad Bolivia, municipio Pedraza estado Barinas.

Así como, Diseñar acciones ambientalistas que contribuyan a la conservación de la cuenca media-alta del río La Acequia, parroquia Ciudad Bolivia, municipio Pedraza estado Barinas, Ejecutar acciones ambientalistas que contribuyan a la conservación de la cuenca media-alta del río La Acequia, parroquia Ciudad Bolivia, municipio Pedraza estado Barinas y Evaluar a través de la opinión de los involucrados los alcances logrados con la aplicación de 
las acciones ambientalistas para la conservación de la cuenca media-alta del río La Acequia, parroquia Ciudad Bolivia, municipio Pedraza estado Barinas.

Del mismo modo, el estudio va a permitir llevar a cabo un trabajo educacional desde al ámbito científico metodológico, puesto que se va a partir de un diagnóstico y la planificación de todas y cada una de las actividades a desarrollarse en el lugar donde ocurre el fenómeno, así como la evaluación de la viabilidad de la aplicación de las mismas. Asimismo, el área ambiental es el principal propósito en cuanto a la recuperación, dado que al lograr la recuperación de dicha cuenca no sólo serán beneficiadas las personas que viven a su alrededor, sino también todas las personas del municipio, ya que este río surte de agua potable a gran parte de la comunidad de Pedraza.

\section{Perspectiva Teórica}

Para dar inicio a los antecedentes se encuentra lbáñez (2012), presenta un trabajo titulado Elaboración de un plan de manejo ambiental para la conservación de la subcuenca del río San Pablo en El Cantón La Maná, provincia de Cotopaxi. Centró su objetivo en elaborar un plan de manejo ambiental para la conservación de la subcuenca del río San Pablo en el cantón La Maná, provincia de Cotopaxi, se enmarcó en una investigación de carácter cualitativo, donde se desplegó un plan de trabajo en función del problema, utilizando la técnica de la observación.

Concluyó que las aguas del río San Pablo presenta un alto grado de contaminación debido a la presencia de excretas generadas por bovinos y porcinos, criaderos ubicados en las riberas del río. Por lo que con el plan de manejo ambiental se reducirá dichos desechos, recuperando los suelos, bajo el control y seguimiento a dicho plan. Recomienda cumplir a cabalidad con todas las actividades planificadas en el plan de manera que haya un mejoramiento en los recursos naturales y en el medio ambiente. 
En tal sentido, el antecedente antes mencionado guarda relación con la investigación, en consideración que se busca mejorar las condiciones ambientales de las cuencas del río La Acequia, mediante actividades educativas que buscan involucrar no sólo a la investigadora sino también a estudiantes jóvenes de la ETAN Don Simón Rodríguez, cuyo propósito es dar un ejemplo cívico que todos deben seguir proyectando.

\subsection{Acciones Ambientales}

La formación ambiental debe partir desde la normativa de la moral que exige la responsabilidad por parte de todos los ciudadanos en cuanto al cuidado del entorno natural, donde principalmente se procure el bienestar entre la sociedad y la naturaleza para que los seres humanos puedan desarrollarse en un ambiente natural cuidado, como una manera de tener mejores condiciones de vida.

Según Sarmiento (2012), corresponde al compromiso de responsabilidad social concreta en la adopción de sistemas de gestión ambiental para garantizar el proceso de producción respetuoso al medio ambiente, de igual manera, utilizar la materia prima natural y renovable mediante procesos reciclables y biodegradables. (pág. 18). En este sentido, las acciones ambientalistas se deben considerar de manera integral, partiendo de lo natural y lo construido, conociendo no sólo los aspectos naturales, tecnológicos, sociales, económicos, políticos, morales, culturales, históricos y estéticos, asumiendo un enfoque interdisciplinario para el tratamiento de la dimensión ambiental, que inspire en el contenido específico.

\subsection{La Conservación}

La conservación es la perpetuación y rehabilitación de los recursos naturales de la tierra: el aire, agua, suelos, plantas, animales y ambiente natural del hombre. La conservación tiene una importancia de primer orden 
para la humanidad. Es la condición necesaria para que ésta pueda sobrevivir; implica un equilibrio dinámico entre el hombre y los recursos naturales, en contraste con la búsqueda del crecimiento económico ilimitado. En su sentido amplio, viene a ser gradualmente un modo de vida. Fuenmayor, (2010), señala en su trabajo que en la III Semana Pro Conservación y Defensa de los Recursos Naturales Renovables organizada por la Universidad de Los Andes en junio de 1961, se presentó una ponencia sobre la enseñanza conservacionista con los siguientes propósitos:

- Formar una conciencia nacional fundada en un profundo respeto por toda vida útil, tendente a la necesaria protección y mejor utilización de los recursos naturales renovables del país.

- Despertar inquietud en los problemas agrarios venezolanos y destacar su influencia en la conservación de los recursos naturales.

- Desarrollar el espíritu de solidaridad y fomentar el sentimiento de cooperación social en relación con los problemas del agro venezolano.

- Impartir orientación vocacional sobre los estudios de las ciencias.

- Capacitar a los educandos, de acuerdo con las características de cada Instituto Educacional, para que puedan participar activamente en las prácticas de conservación y recuperación de los recursos renovables.

La conservación demanda una actitud de respeto a la naturaleza y sus recursos; un reconocimiento de su valor y del valor de su uso apropiado y previsor. El agricultor debe sentirse obligado a dejar el suelo que to ha sostenido en tan buenas o mejores condiciones que como lo encontró.

El público en general, al igual que los técnicos y especialistas debe aceptar que los bosques aptos para la explotación forestal, han de ser utilizados en base a la producción permanente (rendimiento sostenido); así como el ganadero debe sentirse moralmente comprometido para mantener y mejorar la calidad de los pastizales. 


\subsection{Normas Elementales de la Enseñanza de la Conservación}

En relación con las normas elementales de la enseñanza de la Conservación, Según Rodríguez (2013b), plantea que son las siguientes: Primordial responsabilidad de la Enseñanza de la Conservación de la naturaleza y de los recursos naturales, es la de infundir a los educandos la comprensión de las leyes fundamentales de la correlación en la naturaleza, de su equilibrio ecológico, lo cual debe determinar la actitud humana para con el medio ambiente que lo rodea. No tratar los componentes o recursos de la naturaleza, como segmentos aislados sino como partes de un solo sistema unitario. Cuidar que el énfasis en las cosas materiales no oscurezca los superiores valores espirituales de la conservación.

Asimismo, Rodríguez sostiene: hay que tener presente que, aunque las ciencias naturales aportan los conocimientos básicos, los objetivos de la conservación son sociales, humanos; No confundir los medios con los fines. La enseñanza de la conservación se guía hacia la conservación de personas y mentalidades, y no hacia la conservación directa de los recursos. No hay que perseguir un proyecto de conservación por el proyecto mismo, desatendiendo sus consecuencias educacionales. Más importantes que los hechos y las cifras, son los conceptos y las actitudes; los primeros cambian, los últimos deben permanecer firmes, el saber y la cultura comienzan con la comprensión.

\subsection{El manejo de Desechos Sólidos}

Tomando en consideración las ideas de Rodríguez (2013c), el manejo de desechos sólidos, es la gestión de los residuos, la recogida, el transporte, tratamiento, reciclado y eliminación de los materiales de desecho. El término generalmente se refiere, "a los materiales producidos por la actividad humana, $y$, en general, para reducir sus efectos sobre la salud y el medio ambiente" (pág. 28), es decir, que la gestión de los desechos es también llevada a cabo para recuperar los propios recursos de dichos residuos. La gestión de los 
desechos puede implicar estados sólidos, líquidos, gases o sustancias radiactivas, con diferentes métodos y técnicas especializadas para cada uno.

\subsection{Las tres $\mathbf{R}$ - Reducir - Reutilizar y Reciclar}

Según Rodríguez (2013d): Este es el principio de las 3 R - Reducir, Reutilizar y Reciclar. La reducción del volumen de residuos significa automáticamente la reducción del número de camiones de basura en las carreteras, la reducción de residuos en vertederos o incinerados. La reducción de la generación de los desechos (variedad de materiales reciclables, junto con la elección de los productos con pocos envases). La reutilización de los residuos (por ejemplo, el casco de las botellas). El reciclaje de residuos (tratamiento de materiales: el compostaje, papel reciclado, o la transformación en energía: la energía térmica, entre otros.

\subsection{Cuencas Hidrográficas}

Las cuencas hidrográficas amparan una gran variedad de plantas y animales, y brindan muchas oportunidades de esparcimiento al aire libre. Al proteger la salud de nuestras cuencas hidrográficas, se puede preservar y mejorar la calidad de vida. Al respecto, Boschi (2010). Una cuenca hidrográfica es un "área de terreno que drena agua en un punto común, como un riachuelo, arroyo, río o lago cercano. Cada cuenca pequeña drena agua en una cuenca mayor que, eventualmente, desemboca en el océano" (pág. 66). Las cuencas hidrográficas son unidades territoriales en la cual el agua que cae por precipitación se reúne y escurre a un punto común o que fluye toda al mismo río, lago, o mar. En esta área viven seres humanos, animales y plantas, todos ellos relacionados.

\section{Metodológica}

Desde el punto de vista epistemológico y metodológico, la investigación 
corresponde en su naturaleza al enfoque cualitativo, para Martínez (2007), "Es una forma de indagación introspectiva colectiva emprendida por participantes en situaciones sociales con el objeto de mejorar la racionalidad de las prácticas sociales o educativa, así como la comprensión de las mismas" (pág. 5). Los métodos cualitativos, ayudan a interpretar el entorno a través del análisis de lo que dicen, hacen o piensan los protagonistas de la investigación, de allí que, la información debe ser recabada en su ambiente natural e interpretada en su marco contextual.

Por otra parte, el tipo subyace en la Investigación de campo, el cual consiste, de acuerdo con el manual de la UPEL (2016), expresa "El análisis sistemático de problemas en la realidad, con el propósito bien sea de describirlos, interpretarlos y entender su naturaleza y factores constituyentes" (pág. 18). Es decir, que se pueden explicar sus causas y efectos haciendo uso de métodos característicos de cualquiera de los paradigmas o enfoques de la investigación conocidos; además este tipo de investigación permite que los datos de interés son recogidos en forma directa de la realidad; considerándose como datos originales o primarios.

Tomando en cuenta lo expuesto, la investigación se centra en realizar un plan de acción centrado en las necesidades del estudio como es la siembra de árboles y realizar actividades de recolección de desechos sólidos en los alrededores de las cuencas del río La Acequia, con ello se busca construir conocimientos a otras personas partiendo desde la práctica directamente con los implicados en el estudio, el cual está estructurado en aspectos o fases: Sensibilización, capacitación e intercambio de experiencias a través de jornadas de motivación, talleres de orientación, jornadas de limpieza, prácticas de reciclaje, siembra de plantas, entre otros.

Los informantes claves son personas que se seleccionaron, siguiendo ciertos criterios como es el grado de credibilidad, honestidad, moral y sobre todo personas que puedan generar información para la investigación, 
convirtiéndose en una fuente importante de información y a la vez va abriendo el acceso a otras personas y a nuevos escenarios, por tal razón Rodríguez, Gil y García, citado en Olvera y Oliverio (2014), quienes exponen que:

El proceso de selección de informantes no se interrumpe, sino que continúa a lo largo de toda la investigación, valiéndose de estrategias diferentes según el tipo de información que se necesita en cada momento. Como consecuencia, este tipo de selección de informantes también puede definirse como fásico, dado que su desarrollo tiene lugar en más de una etapa o momento de investigación. Y, por ende, se trata de un proceso secuencial, que comienza generalmente asociado con la elección de fenómenos que resultan prioritarios para continuar vinculando al estudio de fenómenos que emergen continuamente a lo largo de la investigación (pág. 6).

Por lo antes descrito, los informantes claves, deben ser tomados mediante un proceso de selección, para el estudio se escogieron tres personas, un funcionario de INPARQUES, dos lugareños que residen en lugar de estudio, que se codificaran como, L1, L2 y L3, para ello los mismos deben reunir las siguientes características, dentro de las cuales se destaca la idoneidad, responsabilidad, credibilidad, ética y sentido de pertenencia de los miembros.

En la investigación propuesta, la entrevista se apoya en un guion de preguntas iniciales y abiertas; del mismo modo, se aplicó la técnica de la observación, con la finalidad de llevar registro de las actitudes de los investigados al momento de ser entrevistados, para ello se usa un cuaderno de notas o diario del investigador e recolección.

\section{Desarrollo de la Entrevista}

Cuadro 1. Entrevistas realizadas a tres (3) informantes claves: Identificados como L1, L2 y L3. 


\section{Temática}

Formación Ambiental

\section{Descripción}

1. ¿Han recibido los lugareños de la zona formación sobre la conservación de las cuencas de los ríos?

L1. Pues para la parte que corresponde a los predios de mi finca se hizo una vez una jornada de reforestación que no tuvo mucho éxito debido al desconocimiento en el aspecto de información sobre del tamaño del trasplante de los árboles, en este caso específicamente del canvil, se sembraron muy pequeño y los bachacos los destrozaron.

L2. Si manejan información de conservación; los habitantes se interesan en la producción para el sustento económico; desconocen las consecuencias del uso indiscriminado de los terrenos adyacentes a las cuencas.

L3. Sí, jornadas de reforestación e información sobre la conservación del agua.

\section{Categoría}

Manejo de información sobre conservación.

Subcategoría

- Desconocimiento de las características de siembra de las plantas a sembrar.

- Uso indiscriminado de los terrenos.

- Jornadas de reforestación.

- Información sobre conservación del agua.

Fuente: Navea (2016).

\subsection{Triangulación de la Entrevista}

De acuerdo a las observaciones realizadas se evidenció que para el manejo de información sobre la conservación de las cuencas de los ríos es deficiente, por cuanto ocasionalmente se han realizado jornadas de reforestación además de incompleta la información referida al tamaño del trasplante de los árboles, sin embargo, los lugareños manejan información de conservación aplicados mediante procedimientos rudimentarios e empíricos el cual tiene como finalidad la producción para el sustento económico y no toman 
en cuenta las consecuencias del uso indiscriminado de los terrenos y la importancia de la conservación del agua.

De los resultados presentados durante la indagación, relacionados con la práctica de actividades agropecuarias indebidas, el uso indiscriminado, la no racionalidad de las personas que han utilizado los terrenos adyacentes a las cuencas con un fin comercial, económico sin pensar en las consecuencias de esas prácticas agropecuarias indebidas. Además del deterioro de la cuenca se debe principalmente a la mala praxis del ser humano y a la forma errónea de la utilización y el aprovechamiento de los espacios, por cuanto un $50 \%$ de su predio convertido en potrero y otro es montaña virgen que sirve de refugio, resguardo, hábitat de recursos hídricos, el cual se ve acechado, por la deforestación tala y quema y a las perforaciones que realizaron los chinos en la parte alta del predio.

Por otra parte, y tomando en cuenta el tipo de acciones de reforestación a tomar en cuenta para rescatar estas cuencas, se destaca la reforestación con especies autóctonas de esa zona a esa altura unos 400 MSNM, beneficiaría el rescate y conservación de la misma, en este caso la cuenca media, asimismo y de forma práctica una estrategia sería la reforestación mediante planes, de cuencas y micro cuencas hídricas, porque estas tributan sus aguas en las cuencas y así mismo ayudan a aumentar su caudal, entonces, rescatar y reforestarla por medio de un plan de seguimiento.

Se destaca en las respuestas dadas por los encuestados referidos a los aportes para el mantenimiento y conservación de las cuencas del rio la Acequia, se destaca el beneficio que proporciona a la producción agropecuaria, también se utiliza para fines turísticos, por otra parte, se deben recoger los desechos que los visitantes en ocasiones dejan tirados para evitar el deterioro de la flora como las orquídeas que se desarrollan en esa área. Por otro lado, las prácticas agronómicas deben ir en armonía con la naturaleza. 
Por tal razón es significativo los aportes de INPARQUES como institución encargada del resguardo de los parques nacionales, monumentos naturales y parques de recreación, de allí, una de las estrategias principales que utiliza el instituto nacional de parques es la sensibilización ambiental de poder formar, orientar y educar a las generaciones y no solo centrar la educación Ambiental a los niños y jóvenes sino a las personas adultas, para evitar el daño ecológico y el resguardo del ambiente.

Se destacó en cuanto al conocimiento y apoyo en las acciones de reforestación, el cambio de la actividad agrícola por especies de árboles para mantener y conservar la cuenca media en caso de ejecutar un plan de reforestación, con la siembra del Canvil, planta autóctona de la cuenca media de esa zona con altura de (400 MSNM), es un arbusto, no muy grande, no requiere de exceso de tierra o arena, tiene la particularidad de que ella, de crecer en un bosque encerrada y logra subirse, son ramas como bejucadas, poco atractivas es conservadora del agua

Finalmente, la importancia de conservar las cuencas significa preservar la vida para todos los habitantes de esta zona, que se benefician a medida que sus aguas van discurriendo, por ello es preocupante la disminución de los caudales de la mayoría de los ríos debido a las malas acciones del hombre principal responsable; por lo que se requiere una práctica continua que favorezca el proceso de cambio para la naturaleza y los recursos que se generen de ella.

\section{Ejecución de las Actividades}

Cuadro 2. Plan de Acción. Acción № 1: Sensibilizar a través de diferentes técnicas de comunicación a los diversos actores sociales e institucionales del programa. Meta: Lograr la sensibilización a través de diferentes técnicas de comunicación a los diversos actores sociales e institucionales del programa. 


\begin{tabular}{|l|}
\hline Estrategias \\
\hline - Técnicas de Comunicación. \\
- Charlas Introductorias, Talleres. \\
\hline Contenidos \\
\hline "Problemática Ambiental Local" \\
"Concienciación y Sensibilización Ambiental" \\
\hline Actividad \\
\hline - Charlas con actores sociales. Socializar principios y normas de la \\
problemática ambiental y la reforestación en la microcuenca. \\
- Selección de espacios de reforestación. \\
- Divulgación, en la comunidad, de los resultados e importancia de la \\
conservación del ambiente. \\
\hline Recursos \\
\hline Humano: \\
Participantes, Facilitador. \\
Instituciones: \\
INIA, MPPAT, MPPAMB, UBV. \\
Material: \\
Rotafolios, Video Beam, Computadora, Marcadores, Pizarra acrílica. \\
Material de apoyo. \\
\hline Tiempo \\
\hline Fecha: 21 y 22 de enero de 2016, 16 horas. \\
\hline Evaluación \\
\hline Instrumento: \\
Acta de asistencia. \\
Criterios: \\
Convocatoria, Reunión con los involucrados. \\
Facilitador: \\
Jeineth del Carmen Navea Rojas \\
\hline
\end{tabular}


fortalecer el ambiente, porque en grupos de trabajo se realizaría una mejor recolección, limpieza y selección de zonas que demandan de la reforestación para desarrollar los proyectos ambientales.

Mediante la evaluación se evidenció que la aplicación de las acciones ambientalistas para la conservación de la cuenca media-alta del río La Acequia, parroquia Ciudad Bolivia, promovió el desarrollo y creatividad en la conservación y reforestación del ambiente, tomando en cuenta la relación que se establece entre la salud y el ambiente. Así como se desarrollarían habilidades y destrezas considerando para cada actividad alternativas posibles en cuanto a los tipos de árboles a sembrar fomentando una conciencia ecologista y autogestión generaría beneficios para la economía de la comunidad.

\section{Recomendaciones}

Realizar una campaña de concientización para el sembrado y mantenimiento de la cuenca mediante Agroforestería de árboles y arbustos en la cuenca media-alta del río La Acequia. Ejecutar de faenas de reforestación conjuntamente con los grupos colaborativos cultivo de árboles que fomente la conservación del ambiente. Participar en charlas frecuentes activas entre los habitantes de la localidad y los organismos competentes del sector La Acequia.

\section{Referencias}

Benetti, G. y Garrido, T. (2012). Comparación de la Comunidad de coleóptero acuáticos, en dos cuencas hidrográficas con distinto grado de acción antropogénica. Universidad de Pontevedra. España.

Boschi, E. (2010). El Ecosistema Estuarial del Río de la Plata. Instituto Nacional de Investigación y Desarrollo Pesquero, Mar del Plata. Argentina. 
Fuenmayor, P. (2010). Informe de Seminario Integrado del 5to año de Ingeniería Forestal. Universidad de los Andes. Mérida. Venezuela. Ibáñez, Y. (2012). Elaboración de un Plan de manejo Ambiental para la Conservación de la Sub Cuenca del río San Pablo en el Cantón La maná, provincia de Cotopaxi. Tesis de Grado previa a la obtención del Título de Ingeniería en medio Ambiente. Universidad Técnica de Cotopaxi. Unidad de Ciencias agropecuarias y Recursos Naturales. Latacunga-Ecuador.

Martínez, M. (2007). Investigación Cualitativa. Editorial Planeta. Caracas.

Olvera, E y Oliverio C. (2014). Manufactura Esbelta y Responsabilidad Social Empresarial. Congreso Internacional de Contaduría, Administración e Informática. Octubre 2014, Ciudad Universitaria. México, D.C.

Rodríguez, J. (2013a,b,c,d). Programa Mundial de Evaluación de los Recursos Hídricos. Lima - Perú.

Rodríguez, M., Gil, D. y García, P. (1999). Metodología de la Investigación Cualitativa. Ediciones ALJIBE México.

Sarmiento, L. (2012). Responsabilidad Social. Editorial Planeta. Caracas. Universidad Pedagógica Experimental Libertador (2016). Manual de Trabajos de Grado de Especialización y Maestría y Tesis Doctorales. 5ta Edición. Caracas. 
Jeineth del Carmen Navea Rojas

e-mail: njeinethdelc@gmail.com

Nacida en Venezuela. Licenciada en Educación Integral,

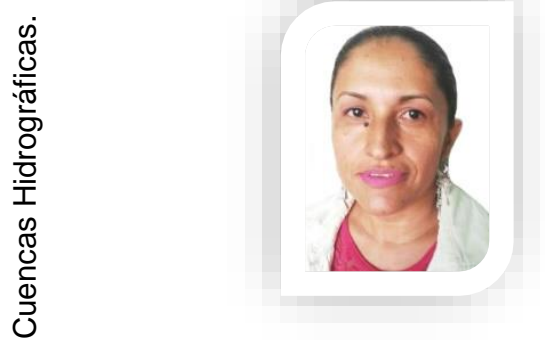

UNELLEZ, Barinas (2006). Cursante de la Maestría en

Educación, Ambiente y Desarrollo, UPEL-IMPM.

Desempeño laboral actual: docente de residencia estudiantil femenina. Experto en Investigación. Manifiesta: interés en formar parte de los árbitros de esta Revista. Línea de investigación: Educación, Ambiente y Desarrollo, área Gestión Ambiental.

El contenido de este manuscrito se difunde bajo una Licencia de Creative Commons ReconocimientoNoComercial-Compartirlgual 4.0 Internacional 